\title{
A Joint User Selection and Resources Optimization Scheduling Algorithm for CoMP
}

\author{
Xincheng Zhong, Weihai Li, Yuqin Lv \\ School of Electronic Engineer, Beijing University of Posts and Telecommunications, Beijing, China \\ Email: zxc072433@gmail.com
}

Received April, 2013

\begin{abstract}
Coordinated Multi-point (CoMP) transmission technology is one of the key techniques in LTE-Advanced, Which can share the channel and data information in multiple cells, and optimize the whole system performance. In order to optimize the average sector throughput and improve the fairness of resource scheduling, a scheduling algorithm based on the resource is mainly investigated. In this algorithm, users in the network are classified firstly and then we combine the fixed resources division and flexible scheduling. System level simulation platform is set up to validate the algorithm and the results turn out that the average throughput is better compared with the traditional scheme.
\end{abstract}

Keywords: CoMP; User Selection; Resource Scheduling; System Level Simulation

\section{Introduction}

As one of the key techniques in LTE-Advanced, CoMP is discussed in detail in the 3GPP. With its effect in reducing interference between neighboring cells, optimizating the whole system performance of multiple cells and expanding the coverage of high data rate, people pay more and more attention to it[1]. Its basic principle is through the coordination of different base transceiver stations (BTS), the interference can be converted to useful signal. According to the information can be shared among multiple transport nodes or not, CoMP technology can be divided into the joint processing (JP) and beam forming[2].

Resource scheduling algorithm for JP is a research hotspot in CoMP to further improve the system performance[3,4], as the CoMP system requires the allocation of resources in multi-point coordinated cells, and single-cell resource scheduling can't meet the requirement of the system scheduling, we need to design scheduling algorithm which can consider interference between adjacent BTSs. The author in [5] has investigated a user-centric scheduling scheme in downlink channel to provide coordination for majority of users, so as to improve the average throughput, but the increasing of the system complexity was not considered. In [6], centralized control in CoMP system was researched and the author proposed to use the MAC layer to transport the information of channel status, so as to reduce the system complexity. In order to avoid the waste of resources and reduce the scheduling complexity, collision avoidance resources scheduling scheme for CoMP was proposed in
[7].

In this paper, the scheduling scheme based on resources for single-user multi-input multi-output (CoMPSU-MIMO) is researched, which improves the traditional scheme of dividing the users into cell-edge users and cell-center users by the SINR values[8], and 2 or 3 sectors coordination is added to reduce the waste of resources, at the same time, on the basis of the scheme to divide the frequency into fixed two parts, resources optimization is considered in one part to ensure profit both edge and center users. We also set up a system level simulation platform and the simulation results also verify the effectiveness of the proposed algorithm.

The rest of paper is organized as follows: In section II, the system model of JP in CoMP is described. Section III introduces the specific scheduling algorithm and the simulation results are showed in section IV. Then conclusions are drawn in section $\mathrm{V}$.

\section{CoMP JP System Model}

The downlink of a cellular network with $M$ hexagonal cells is considered and each cell is partitioned into 3 sectors with $K$ active users served within the coverage of each sector. The total number of BTSs in the system is $M$. Each BTS which corresponds to one sector is equipped with $N_{t}$ transmit antennas, while each user has $N_{r}$ receive antennas. We assume each three adjacent sectors as a cluster[9], show as in Figure 1.

1) Considering the serving BTS $m$, and user $k$ in cluster $c$ severed in the CoMP mode, the received signal is given as: 


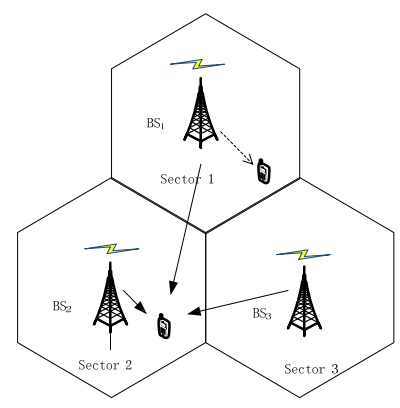

Figure 1. The system model of CoMP JP.

$$
\begin{aligned}
y_{k}(t)= & H_{m, k}(t) w_{k} s_{k}(t)+\sum_{\substack{n \in C \\
n \neq m}} H_{n, k}(t) w_{k} s_{k}(t) \\
& +\sum_{j \in \gamma} H_{j, k}(t) w_{j} s_{j}(t)+N_{0}
\end{aligned}
$$

where the first item in the right-hand-side(RHS) of equation(1) presents the transmission signal of BTS $m, H_{m, k}(t)$ denotes the $N_{r} \times N_{t}$ channel matrix from BTS $m$ to user $k$, $s_{k}(t)$ denotes the signal from BTS $m$ to user $k, w_{k}$ presents the precoding matrix of $s_{k}(t)$. The second item presents the coordinated signal from the BTSs of cluster $c$, $H_{n, k}(t)$ denotes the channel matrix form other BTSs in cluster $c$ to user $k$. The third item presents the interference signal from the BTSs out of the cluster $c$ to user $k$.

Assuming that each BTS has perfect channel state information(CSI) of all the users in the sector coverage, and BTS within the same cluster can fully share CSI and user data, the SINR of user $k$ is given as:

$$
\operatorname{SINR}=\frac{p_{k}\left|H_{m, k} w_{m}\right|^{2}+\sum_{n \in C, n \notin m} p_{n}\left|H_{n, k} w_{n}\right|^{2}}{N_{0}+\sum_{j \in \gamma} p_{j}\left|H_{j, k}(t) w_{j}\right|^{2}}
$$

where $p_{k}$ presents the transmit power from BTS $m$ to user $k, p_{n}$ is the transmit power from other BTSs in cluster $c$ to user $k$, and $p_{j}$ presents the interference power from BTSs out of cluster $c$ to user $k$.

2) Considering user $k$ in cluster $c$ severed in the NoCoMP mode, and the serving BTS is $m$, then the received signal is given as:

$$
\begin{aligned}
y_{k}(t)= & H_{m, k}(t) w_{k} s_{k}(t)+\sum_{\substack{n \in C \\
n \neq m}} H_{n, k}(t) w_{n} s_{n}(t) \\
& +\sum_{j \in \gamma} H_{j, k}(t) w_{j} s_{j}(t)+N_{0}
\end{aligned}
$$

where the second item is not the coordinated signal but the interference signal from BTSs of the cluster $c$ to user $k$, then the SINR of user $k$ is given as:

$$
\operatorname{SINR}_{K}=\frac{p_{k}\left|H_{m, k} w_{m}\right|^{2}}{N_{0}+\sum_{j \in \gamma} p_{j}\left|H_{j, k}(t) w_{j}\right|^{2}+\sum_{n \in C, n \neq m} p_{n}\left|H_{n, k} w_{n}\right|^{2}}(4)
$$

where presents the transmit power from BTS m to user $k$, is the interference power from other BTSs in cluster $c$ to user $k$, and $p_{i}$ presents the interference power from BTSs out of cluster $c$ to user $k$.

\section{The Proposed Scheduling Algorithm}

\subsection{User Selection}

Proposed in [8], the network is divided into a number of disjoint clusters, where each cluster contains 3 adjacent sectors, and all of the users are divided into cell-center users and cell-edge users according to the SINR, which work in the No-CoMP and CoMP mode respectively. When in CoMP mode, all the three sectors in the cluster send signals to one user at the same time. But fixed threesectors coordination can't guarantee the best scheduling of resources, as shown in Figure 2, when the channel situation is not good enough between the user and the BTS, the sector of the BTS is not suitable to coordinate with the adjacent sectors. Considering this situation, twosectors CoMP mode is added.

1) three-sectors CoMP user: According to the equations (2) and (4), we can describe the SINR of user $k$ working in No-CoMP and CoMP mode as SINR $_{\text {no-CoMP }}$ and SINR $_{\text {three-CoMP, then user } k \text { works in three-sectors }}$ CoMP mode if

$$
\text { SINR }_{\text {three-CoMP }} \geq 3 \times \operatorname{SINR}_{\text {No-CoMP }}
$$

2) two-sectors CoMP user: According to the equation (2), considering user $k$ works in CoMP mode with two sectors which contain better channel gain in the cluster, we can describe the SINR of user $k$ as $\operatorname{SINR}_{t w o-C o M P}$, then user $k$ works in two-sectors CoMP if

$$
3 \times \operatorname{SINR}_{\text {no-СoMP }}>\operatorname{SINR}_{\text {two-CoMP }} \geq 2 \times \operatorname{SINR}_{\text {no-CoMP }} .
$$

3) cell-center user: Other users are cell-center users which work in No-CoMP mode.

\subsection{Resources Optimization Scheduling}

In traditional scheduling scheme, the frequency band is divided into two parts, one for CoMP mode and the other for No-CoMP mode. PF scheduling algorithm is implemented separately in each frequency zone for different

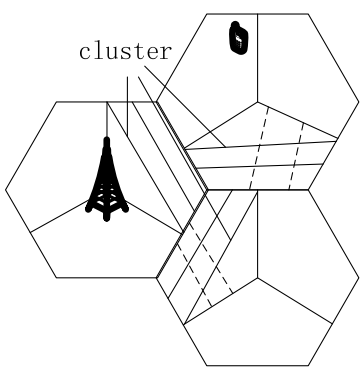

Figure 2. An example of clusterd network. 
work mode. However, this scheme can hardly guarantee the fairness within the system while improving the system throughput at the same time. In addition, the traditional schemes arrange the CoMP frequency zone at the beginning of the whole frequency band, which may not be the best RBs to cell-edge UEs for CoMP-SU-MIMO transmission. A kind of resources optimization scheduling scheme is proposed in this paper, The basic idea is the combining of the fixed partition and flexible scheduling of resources. One part of the resources are used to ensure the demand of the cell-center users and in the other part, starting from each resource block(RB), the most appropriate way of scheduling is selected. In this way, cell-center users which are the larger part can get sufficient resources and at the same time, the cell-edge users can also get the most appropriate scheduling resources. System performance and fairness is balanced and the main steps are as follows:

1) For all the RBs in the network, we take a specific proportion to be used for the cell-center users, as shown in equation (7), $\beta$ is the ratio of factors which can set different values according to the state of channel, so that the fixed RBs used for the cell-center users in different clusters can be adjusted according to the different load.

$$
\alpha=\frac{\operatorname{Num}_{\text {No-CoMP }}}{\text { Num }_{\text {No-CoMP }}+N_{\text {three-CoMP }}+N u m_{\text {two-CoMP }}} \times \beta
$$

2) Assume the set of all the cell-edge users in the coordination cluster is $\Omega_{c}$, the set of cell-center users in sector 1 is $\Omega_{1}$, the set of cell-center users in sector 2 is $\Omega_{2}$, and the set of cell-center users in sector 2 is $\Omega_{3}$.

3) The set of fixed RBs which are allocated to the cell-center users is $\phi_{1}$, and the other flexible RBs are in the set of $\phi_{2}$.

4) RBs in $\phi_{1}$ are selected for cell-center users, according to the PF scheduling algorithm, for the RBs in $\phi_{2}$, calculate the highest priorities $p_{c} 、 p_{1} 、 p_{2} 、 p_{3}$ of the users in $\Omega_{c} 、 \Omega_{1} 、 \Omega_{2} 、 \Omega_{3}$ respectively, and the selected users are $k, j_{1}, j_{2}, j_{3}$.

5) We compare them as follows

$$
3 p_{c} p_{<} p_{1}+p_{2}+p_{3}
$$

If the left-hand-side of (8) is no less than the right-hand-side, the system will perform the CoMP mode in the $\mathrm{RB}$ and user $k$ is scheduled for transmission through BTSs coordination with two or three sectors; otherwise, the three BTSs of cluster $c$ will operate single sector No-CoMP transmissions for users $j_{1}, j_{2}, j_{3}$ independently.

6) The schedule of RBs is completed, and if all the RBs are allocated, the schedule is over, otherwise, $a=a+1$, the RB continues to be scheduled. The flow chart of the proposed scheme is as Figure 3.

\section{Simulation Results}

In this section, the performance of the proposed algorithm is evaluated through our system level simulation. A cellular system with 19 hexagonal cells is assumed, and the distance between base stations is $500 \mathrm{~m}$. The channel bandwidth is $10 \mathrm{Mhz}$ with $50 \mathrm{RBs}$ and each one is 180 khz with 12 subcarriers. We choose the number of antennas to be $N_{\mathrm{t}}=N_{\mathrm{r}}=2$. Each BTS has perfect CSI of all the users in the sector, and BTSs within the same cluster an fully share CSI and user data.

Figure 4 compares the normalized user throughput CDF of Round Robin No-CoMP and resources optimization CoMP, and the result illustrates the use of CoMP can greatly improve user throughput in hexagonal cells with the coordination of the different base stations.

Figure 5 compares the normalized user throughput CDF of three different CoMP scenarios which are Round Robin CoMP, the traditional fixed frequency CoMP and the resources optimization CoMP. The result illustrates, due to the resources optimization algorithm, the majority cell-center users can get fixed resources and at the same time, each other RB is able to choose the optimal scheduling according to channel state, the throughput of the users is effectively improved and resource utilization is optimized.

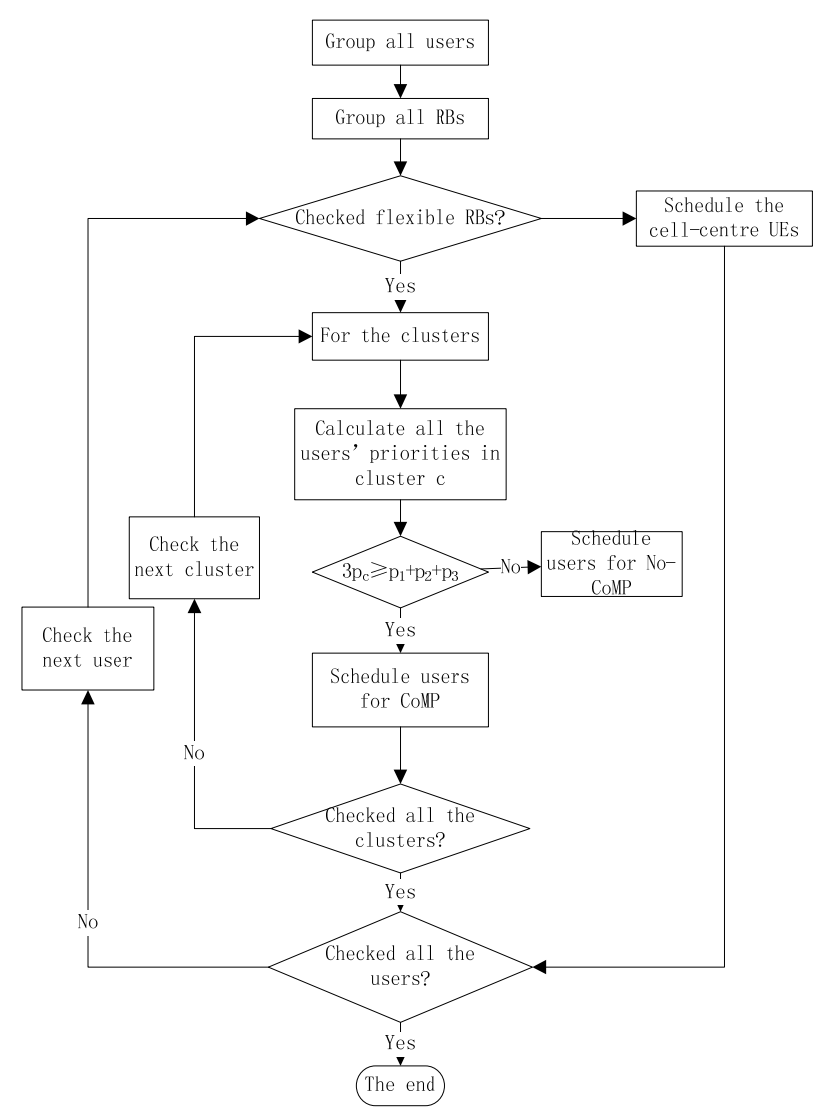

Figure 3. The flow chart of the proposed scheme. 


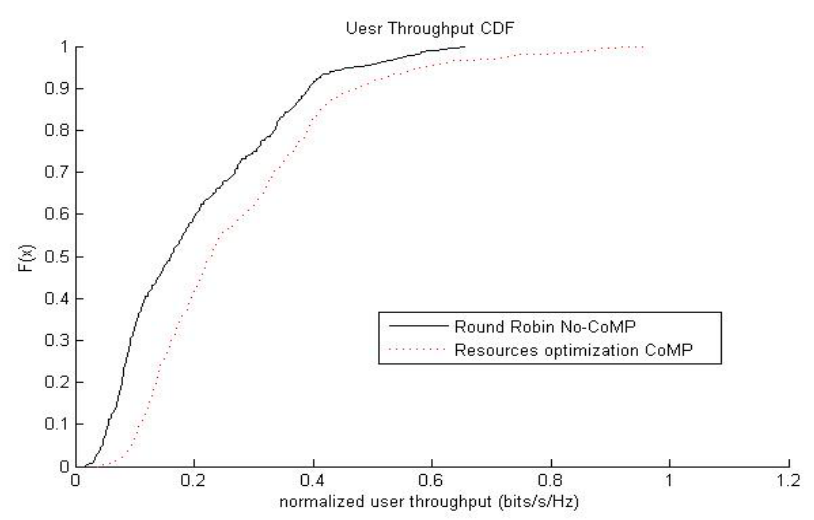

Figure 4. CDF of user throughput for CoMP and No-CoMP.

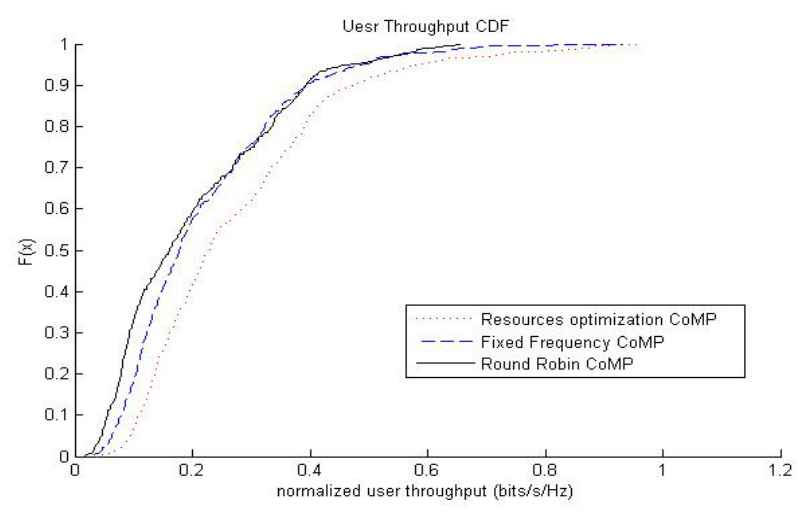

Figure 5. CDF of user throughput for different CoMP systems.

\section{Conclusions}

A joint user selection and resources optimization scheduling algorithm for CoMP is proposed in this paper, the algorithm adds the flexible two or three sectors coordination in user selection at the same time, the scheme of resources allocation is improved. By the system level simulation, the algorithm is compared with the NoCoMP scenario and other two CoMP scenarios, and the results verify the effectiveness of the proposed algorithm.

\section{Acknowledgements}

This paper is supported by Beijing Municipal Science \& Technology Commission (Grant No. Z111100066511007) and Fundamental Research Fund for the Central Universities(2013RC0203).

\section{REFERENCES}

[1] 3GPP TS 36.814, "Further Advancement for E-UTRA Physical Layer Aspects,” 2009.

[2] 3GPP TR 36.819, "Coordinated Multi-point Operation for LTE Physical Layer Aspects,” 2011-2012.

[3] G. Q. Li and H. Liu, "Downlink Radio Resource Allocation for Multi-cell OFDMA System,” IEEE Transactions on Wireless Communications, Vol. 5, No. 12, 2006, pp. 3451-3459.doi:10.1109/TWC.2006.256968

[4] X. Chen, X.-D. Xu, H.-J. Li and X.-F. Tao, T. Svensson and C. Botella, "Improved Resource Allocation Strategy in SU-CoMP Network," The Journal of China Universities of Posts and Telecommunications, Vol. 18, 2011, pp. 7-12.

[5] K. Zang, Y. F. Zheng, M. R. He and Z.-J. Jiang, "A Survey on User-centric Cooperative Set Selection in CoMP," Journal of Lanzhou Jiaotong University, Vol. 31, 2012, pp. 131-134.

[6] S. Brueck, L. Zhao and J. Giese, “Centralized Scheduling for Joint Transmission Coordinated Multi-point in LTE-Advancd,” WSA, 2010, pp. 177-184.

[7] W.-A. Zhou, Z.-Y. Tan, W. Chen, S. Chen and Z.-J. Ren, "Collision Avoidance Resources Scheduling Scheme for CoMP," The Journal of China Universities of Posts and Telecommunications, Vol. 35, 2013, pp. 86-89.

[8] B. B. Wang, B. B. Li and M. Q. Liu, “A Novel Precoding Method for Joint Processing in CoMP,” International Conference on Network Computing and Information Security, Vol. 1, 2011, pp. 126-129.

[9] Z. Jun, C. Runhua, J. G. Andrews, A. Ghosh and R. W. Heath, "Networked MIMO with Clustered Linear Precoding," Wireless Communications, IEEE Transaction on, Vol. 8, 2009, pp. 1910-1921. doi:10.1109/TWC.2009.080180 